\title{
Effects of photodynamic therapy for superficial esophageal squamous cell carcinoma in vivo and in vitro
}

\author{
KAORU KAWAZOE ${ }^{1}$, HAJIME ISOMOTO ${ }^{1}$, NAOYUKI YAMAGUCHI ${ }^{1}$, NAOKI INOUE ${ }^{1}$, \\ RYOHEI UEHARA ${ }^{1}$, KAYOKO MATSUSHIMA ${ }^{1}$, TATSUKI ICHIKAWA ${ }^{1}$, FUMINAO TAKESHIMA ${ }^{1}$, \\ TAKASHI NONAKA ${ }^{2}$, ATSUSHI NANASHIMA ${ }^{2}$, TAKESHI NAGAYASU $^{2}$, \\ MASATAKA UEHARA ${ }^{3}$, IZUMI ASAHINA ${ }^{3}$ and KAZUHIKO NAKAO ${ }^{1}$ \\ ${ }^{1}$ Department of Gastroenterology and Hepatology, ${ }^{2}$ Division of Surgical Oncology, \\ Department of Translational Medical Sciences, and ${ }^{3}$ Department of Regenerative Oral Surgery, \\ Nagasaki University Graduate School of Biomedical Sciences, Nagasaki 852-8501, Japan
}

Received June 8, 2010; Accepted July 21, 2010

DOI: 10.3892/ol_00000155

\begin{abstract}
Photodynamic therapy (PDT) is an ablative treatment leading to intracellular photoexcitation and injury. A total of 15 patients with superficial esophageal squamous cell carcinoma (ESCC) without metastasis underwent PDT and 48-72 $\mathrm{h}$ after intravenous Photofrin, the patients were treated with a $630-\mathrm{nm}$ excimer dye laser. A total of 13 patients had local tumor recurrence after definitive chemoradiotherapy (CRT) consisting of 5-fluorouracil (5-FU) and cisplatin (CDDP). Of 6 patients, 5 had submucosal ESCC and were treated with S-1. Complete reponse was achieved by 11 patients with initial PDT, but 2 had recurrences. The recurrent/residual tumors were successfully treated with repeated PDT. Two patients with intramucosal ESCC succumbed due to metastatic disease, but 11 patients were disease-free. The 5 patients treated with S-1 remained alive despite submucosal ESCC. PDT was applied to human ESCC cells in vitro in the presence or absence of 5-FU or CDDP. The combination of PDT with 5-FU or CDDP resulted in enhanced cytotoxic effects, thereby reducing the effective dosage of each drug. PDT is a promising treatment option for selected ESCC cases, particularly for local recurrence following CRT. Our experience suggests that PDT is more effective when combined with chemotherapy.
\end{abstract}

\section{Introduction}

Definitive chemoradiotherapy (CRT) is considered to be an alternative, standard, non-surgical treatment for esophageal cancer, since it shows comparable clinical outcomes to esophagectomy

Correspondence to: $\mathrm{Dr}$ Hajime Isomoto, Department of Gastroenterology and Hepatology, Nagasaki University Graduate School of Biomedical Sciences, 1-7-1 Sakamoto, Nagasaki 852-8501, Japan

E-mail: hajimei2002@yahoo.co.jp

Key words: photodynamic therapy, esophageal squamous cell carcinoma, chemoradiotherapy, 5-fluorouracil
$(1,2)$. However, persistent or recurrent locoregional disease commonly occurs after CRT and remains an unresolved issue $(1,2)$. The survival of patients who do not achieve complete response (CR) is dismal (3). Therefore, improvement of local control is one of the major factors for better survival of esophageal cancer patients who are treated with CRT. Salvage esophagectomy has been a curative treatment of choice, but it is a more difficult procedure than primary esophagectomy, and the postoperative mortality rates are relatively higher (4). Moreover, there are no curative chemotherapy protocols currently available for the treatment of residual esophageal tumors. Again, salvage endoscopic mucosal resection (EMR) is used for locoregional disease without distant metastasis primarily or following CRT failure (5). Nevertheless, EMR has limitations with respect to resection size and is difficult to perform in cases with fibrous scar lesions (6,7). Endoscopic submucosal dissection (ESD) allows for the removal of larger gastrointestinal tumors en bloc, but advanced skill is required. ESD is also associated with a substantial risk of complications $(8,9)$.

Photodynamic therapy (PDT) is an ablative treatment for rapidly proliferating tissues, including dysplastic and malignant lesions (1). It involves administration of a photosensitizing drug followed by the application of a specific wavelength of light, leading to intracellular photoexcitation and injury (11). Theoretically, PDT can cure gastrointestinal neoplasms contained within the submucosal layer $(10,12,13)$. It is a highly effective, painless, and usually well-tolerated procedure that is simple to perform $(10,13)$. Unlike resection, PDT has the advantage of preserving the integrity of the esophagus. This study aimed to investigate PDT as definitive management for non-metastatic superficial esophageal squamous cell carcinoma (ESCC). In addition, the in vitro study and our case series suggest that PDT is more effective for ESCC when combined with chemotherapy, such as fluorouracil.

\section{Patients and methods}

Clinical setting of PDT. Between April 2007 and March 2010, 15 patients with ESCC were treated by PDT at Nagasaki 
University Hospital. Although all persistent or recurrent tumors were surgically resectable, the decision to undergo non-surgical treatment was based on the patients' refusal of surgery or severe concomitant disease. The criteria for PDT were: i) lack of detection of lymph node or distant metastases; ii) the ESCC tumor invasion was within the mucosal and/ or submucosal layer on endoscopic ultrasonography; iii) other non-surgical treatments including EMR and ESD were not indicated for reasons of difficulty or non-curability; and iv) written informed consent was obtained from each patient. A total of 13 patients had CRs with CRT, but the tumor was recurrent at the primary site. In addition, there were 2 naïve cases of ESCC. The CRT consisted of $60 \mathrm{~Gy}$ irradiation, along with 2 cycles of continuous infusion with 5 -fluorouracil (5-FU) and cisplatin (CDDP). 5-FU $\left(700 \mathrm{mg} / \mathrm{m}^{2}\right.$, 24-h intravenous infusion) was administered on days 1 to 4 . CDDP $\left(70 \mathrm{mg} / \mathrm{m}^{2}\right.$, 2-h intravenous infusion) was administered with hydration on day 1 . This schedule was repeated twice, every 4 weeks. Radiotherapy was initiated concurrently on the first day of the first and second course of chemotherapy and was delivered in 30 fractions of $2 \mathrm{~Gy}$ for a total of $60 \mathrm{~Gy}$. In addition, 2 courses of the same chemotherapy were added. The definition of CR after CRT was: i) disappearance of the tumor lesion or ulcer of the primary site with confirmed cancer-negative histology, and ii) disappearance of measurable or assessable metastatic lesions confirmed on computed tomography (CT) (14).

The PDT procedure began with an intravenous administration of $2 \mathrm{mg} / \mathrm{kg}$ of Photofrin (Wyeth, Tokyo, Japan), followed by dye laser irradiation. The $630-\mathrm{nm}$ wavelength laser beam was provided by an excimer dye laser (EDL-1; Hamamatsu Photonics, Hamamatsu, Japan). The laser treatment was performed 48 and/or $72 \mathrm{~h}$ after injection of the photosensitizer. The laser was delivered via a free-cut fiber introduced into the operative channel of the fiberscope. The distal tip of the fiber was kept $\sim 1 \mathrm{~cm}$ from the surface of the lesion. The total light density was $80 \mathrm{~J} / \mathrm{cm}^{2}$, with a $4-\mathrm{mJ} /$ pulse maximum pulse energy and a $40-\mathrm{Hz}$ pulse frequency. All of the patients were instructed to avoid direct exposure to sunlight for 4 weeks after the injection. Endoscopic examination with biopsy was repeated 7 days later, at 1, 3, 6 and 12 months after PDT, and then annually. The effectiveness of PDT was classified as CR when there was no macroscopic or microscopic evidence of ESCC, or non-CR when a tumor was observed at endoscopy and confirmed histologically. Local recurrence was defined as a relapse after achieving CR (14). Cervical/thoracic/abdominal CT was performed at 3, 6 and 12 months after PDT and then annually. Blood samples were obtained from each patient before and 7 days after PDT for measurement of serum total reactive oxygen species (ROS) to monitor whether the total ROS values could predict the efficacy of PDT (15).

For patients with submucosal ESCC, $50 \mathrm{mg}$ of S-1 (Taiho Pharmaceutical, Tokyo, Japan), an oral fluorouracil, was administered twice daily for 28 consecutive days, followed by 14 days of rest for 12 months. S- 1 consists of a 1:0.4:1 molar ratio mixture of tegafur and two modulating substances: gimeracil (5-chloro-2,4-dihydroxypyrimidine; CDHP) and oteracil (potassium oxonate) (16).

In vitro study. The cytotoxic effect of combination treatment with PDT and 5-FU or CDDP on a human ESCC cell line,
OE21, was investigated. OE21 cells were obtained from the American Type Culture Collection (Manassas, VA, USA) and grown in RPMI-1640 (Nissui Ceutical, Tokyo, Japan) with $10 \%$ fetal bovine serum, glutamine $(0.6 \mathrm{mg} / \mathrm{ml})$, penicillin $(100 \mathrm{U} / \mathrm{ml})$ and streptomycin $(100 \mathrm{mg} / \mathrm{ml})$ at $37^{\circ} \mathrm{C}$ in a humidified atmosphere of $5 \% \mathrm{CO}_{2}$ in air. OE21 cells were exposed to 5-FU (Sigma Aldrich, St. Louis, MO, USA) or CDDP (Nippon Kayaku, Tokyo, Japan) at various concentrations for $24 \mathrm{~h}$ to set the half maximal $(50 \%)$ inhibitory concentration $\left(\mathrm{IC}_{50}\right)$ for each chemotherapy drug. Subsequently, the cells were exposed to Photofrin at various concentrations in the presence of CDDP or 5-FU at the selected $\mathrm{IC}_{50}$ for each drug for $24 \mathrm{~h}$, followed by irradiation with an Nd:YAG-pumped dye laser $\left(630 \mathrm{~nm}, 5.0 \mathrm{~J} / \mathrm{cm}^{2}\right)(17)$ (Quanta-Ray ${ }^{\circledR}$ DCR-3 and PDL-2, Spectra Physics, Mountain View, CA, USA).

Cellular survival was assessed by the 3-(4,5-dimethylthiazol-2-yl)-5-(3-carboxymethoxyphenyl)-2-(4-sulfophenyl)-2H-tetrazolium (MTS) assay (Promega, Madison, WI, USA). OE21 cells were plated at a density of $3 \times 10^{3}$ cells/well on 96-well plates and grown overnight. The assay was initiated $24 \mathrm{~h}$ later by adding $20 \mu \mathrm{l}$ of MTS solution reagent to $100 \mu \mathrm{l}$ of culture medium for each well. After incubation for $3 \mathrm{~h}$ at $37^{\circ} \mathrm{C}$, the plates were read in a microplate autoreader (Molecular Devices, Sunnyvale, CA, USA) at a wavelength of $490 \mathrm{~nm}$. The results were expressed as the mean optical density for selected paradigms performed in duplicate. All assays were set up in triplicate, and the results were expressed as the means \pm standard deviation (SD). Statistical significance was determined by the unpaired Student's t-test using the statistical package StatView (Abacus Concepts, Inc., Berkeley, CA, USA). P<0.01 was considered to be significant.

\section{Results}

Clinical outcomes. Patient baseline characteristics prior to PDT are summarized in Table I. The median age was 71 years (range 54-86). There were 14 males and 1 female. Of the 15 patients, 9 had intramucosal ESCC, and the remaining patients had submucosally invasive ESCC. Clinical outcomes with PDT are summarized in Table II. The median total light dosage delivered was $600 \mathrm{~J}$ (range 280-1065). Patient tumors showed a response to PDT. CR was achieved in 11 (73\%) of the 15 patients, but 2 patients experienced local recurrence after PDT. Thus, the 6 patients were treated again with PDT, and the recurrent/ residual lesions were eliminated in all 6 cases. Initial PDT was successfully performed in 4 of the 6 submucosal ESCC cases (Fig. 1), while CR was achieved in 7 of the 9 mucosal tumor cases. Otherwise, no clinicopathological factors had a significant impact on PDT outcomes. At a median follow-up period of 23 months (range 4-35), 13 patients were alive, and 11 were disease-free. Two patients with intramucosal ESCC succumbed due to metastatic disease despite having no local recurrence in the irradiated esophagus. The 5 patients treated with S-1 were alive, and 3 were disease-free, although the median follow-up period was 8 months. One patient with submucosal ESCC was unable to commence chemotherapy treatment due to sustained bone marrow suppression following CRT.

No significant difference was found in pretreatment serum total ROS levels between the CR (156.3 \pm 36.2 units) and nonCR groups (177.0 \pm 28.0 units) at the initial PDT. No significant 
Table I. Background characteristics of the patients with superficial esophageal squamous carcinoma treated by photodynamic therapy (PDT).

\begin{tabular}{|c|c|c|c|c|c|c|}
\hline Case no. & Age & Gender & Esophageal location & Endoscopic findings & Invasive depth & Chemoradiation \\
\hline 1 & 73 & M & Thorax & IIc & sm & Recurrence \\
\hline 2 & 54 & M & Thorax & III & $\mathrm{sm}$ & Recurrence \\
\hline 3 & 85 & M & Thorax & IIc & $\mathrm{m}$ & Recurrence \\
\hline 4 & 71 & M & Cervix & $\mathrm{IIb}$ & $\mathrm{m}$ & Recurrence \\
\hline 5 & 75 & M & Cervix & $\mathrm{IIb}$ & $\mathrm{m}$ & Recurrence \\
\hline 6 & 69 & $\mathrm{M}$ & Abdomen & IIa & $\mathrm{sm}$ & Recurrence \\
\hline 7 & 59 & $\mathrm{M}$ & Thorax & $\mathrm{IIb}$ & $\mathrm{m}$ & Recurrence \\
\hline 8 & 76 & M & Abdomen & IIc & $\mathrm{m}$ & Recurrence \\
\hline 9 & 79 & $\mathrm{M}$ & Thorax & $\mathrm{I}$ & $\mathrm{sm}$ & Recurrence \\
\hline 10 & 69 & M & Thorax & IIc & $\mathrm{m}$ & Recurrence \\
\hline 11 & 70 & M & Thorax & IIb & $\mathrm{m}$ & Recurrence \\
\hline 12 & 59 & $\mathrm{M}$ & Thorax & IIb & $\mathrm{m}$ & Naïve \\
\hline 13 & 60 & $\mathrm{~F}$ & Cervix & $\mathrm{IIb}$ & $\mathrm{m}$ & Recurrence \\
\hline 14 & 86 & $\mathrm{M}$ & Thorax & IIc & $\mathrm{sm}$ & Recurrence \\
\hline 15 & 76 & $\mathrm{M}$ & Thorax & IIc & $\mathrm{sm}$ & Naïve \\
\hline
\end{tabular}

sm, submucosal; m, mucosal; M, male; F, female.

Table II. Clinical outcomes of photodynamic therapy.

\begin{tabular}{|c|c|c|c|c|c|c|c|}
\hline $\begin{array}{c}\text { Case } \\
\text { no. }\end{array}$ & $\begin{array}{c}\text { PDT } \\
\text { dose }(\mathrm{J})\end{array}$ & Complications & $\begin{array}{l}\text { Response to } \\
\text { initial PDT }\end{array}$ & $\begin{array}{l}\text { Repeated } \\
\text { PDT }\end{array}$ & $\begin{array}{c}\text { Chemotherapy } \\
\text { with PDT }\end{array}$ & $\begin{array}{l}\text { Follow-up } \\
\text { (months) }\end{array}$ & Prognosis \\
\hline 1 & 400 & High-grade fever, chest pain & $\mathrm{CR}$ & & & 35 & Alive \\
\hline 2 & 480 & Chest pain & Non-CR & Performed & S-1 & 33 & Alive \\
\hline 3 & 620 & High-grade fever & Non-CR & Performed & & 33 & Alive \\
\hline 4 & 940 & High-grade fever & $\mathrm{CR}$ & & & 31 & Deceased \\
\hline 5 & 355 & Esophageal stenosis & $\mathrm{CR}$ & & & 30 & Alive \\
\hline 6 & 1065 & Skin phototoxicity & $\mathrm{CR}$ & & S-1 & 28 & Alive \\
\hline 7 & 520 & Chest pain, high-grade fever & $\mathrm{CR}$ & & & 23 & Deceased \\
\hline 8 & 540 & Chest pain, high-grade fever & $\mathrm{CR}$ & & & 23 & Alive \\
\hline 9 & 900 & Esophageal stenosis & Non-CR & Performed & S-1 & 9 & Alive \\
\hline 10 & 600 & High-grade fever, mediastinitis & $\mathrm{CR}$ & & S-1 & 8 & Alive \\
\hline 11 & 400 & Esophageal stenosis & Non-CR & Performed & & 8 & Alive \\
\hline 12 & 1000 & None & Recurrence after CR & Performed & & 7 & Alive \\
\hline 13 & 280 & None & $\mathrm{CR}$ & & & 4 & Alive \\
\hline 14 & 740 & High-grade fever, mediastinitis & Recurrence after CR & Performed & & 4 & Alive \\
\hline 15 & 860 & None & $\mathrm{CR}$ & & S-1 & 33 & Alive \\
\hline
\end{tabular}

CR, complete response; PDT, photodynamic therapy.

difference was noted in the 7-day post-treatment total ROS values irrespective of the treatment outcome $(205.2 \pm 56.5$ and 204.7 \pm 27.2 units, respectively). When limited to patients who had successful PDT, the serum total ROS values were significantly increased from $156.3 \pm 36.2$ units before PDT to $205.2 \pm 56.5$ units after PDT $(\mathrm{p}<0.05)$.

In all cases, Photofrin administration was well tolerated. There were no allergic reactions or injection site irritation. As for acute complications within 7 days after PDT, high fever $\left(>38.0^{\circ} \mathrm{C}\right)$ and chest pain that required analgesic treatment were observed in 10 and 4 patients, respectively. A total of 6 patients experienced significant complications: 2 had mediastinitis; 3 had esophageal stenosis that required repeated endoscopic balloon dilation; and 1 had cutaneous phototoxicity. Each complication was successfully managed with medical treatment. No deaths were attributable to the PDT procedure itself. 
A

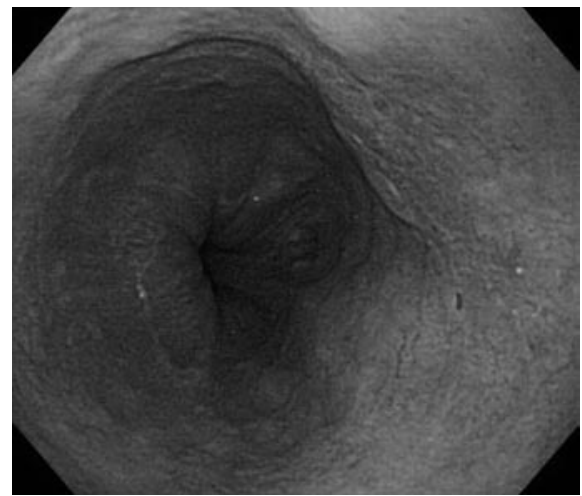

C

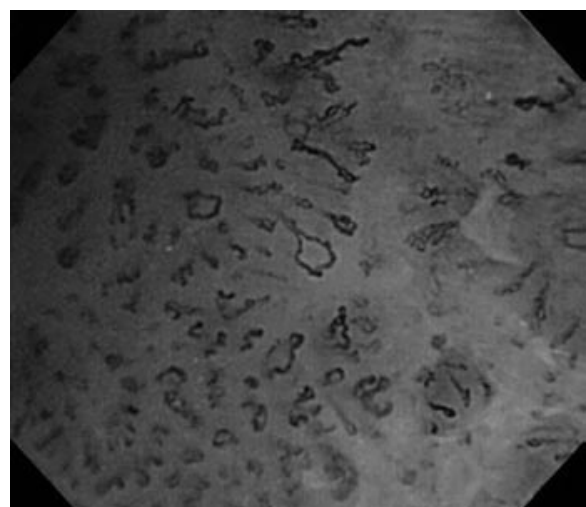

$\mathbf{E}$

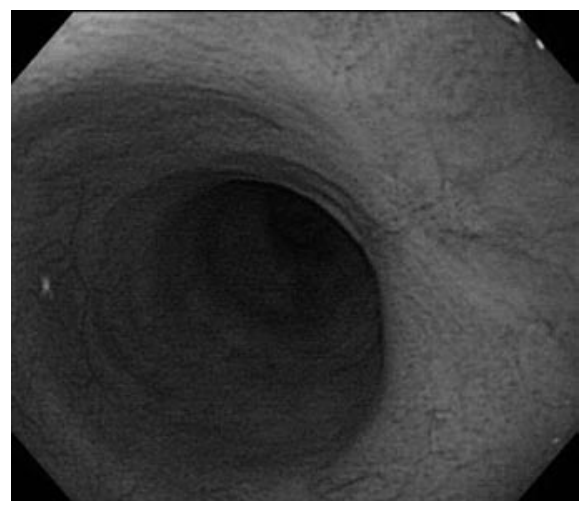

G

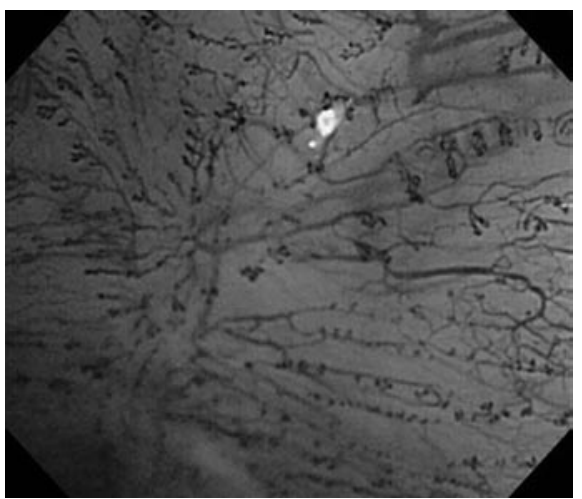

In vitro study. The two chemotherapeutic agents substantially affected survival of the OE21 cells with the $\mathrm{IC}_{50}$ of single 5-FU and CDDP set at 3.5 and $9.0 \mu \mathrm{M}$, respectively. PDT showed clear cytotoxic activity against the OE21 cells. To induce $\mathrm{IC}_{50}$, a Photofrin concentration of $10 \mu \mathrm{g} / \mathrm{ml}$ was required for this ESCC cell line at a laser power of $5 \mathrm{~J} / \mathrm{cm}^{2}$. Following the combination of $3.5 \mu \mathrm{M}$ of 5-FU with PDT using Photofrin at various concentrations, a significant synergistic
B

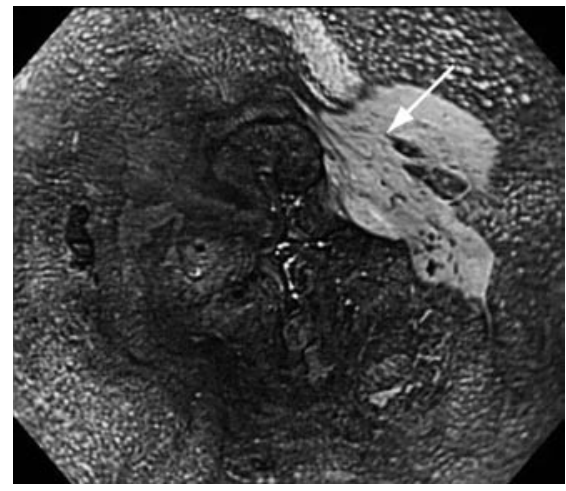

D

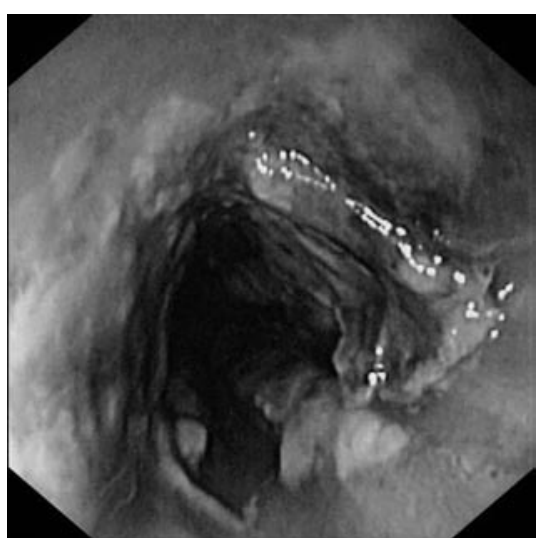

$\mathbf{F}$

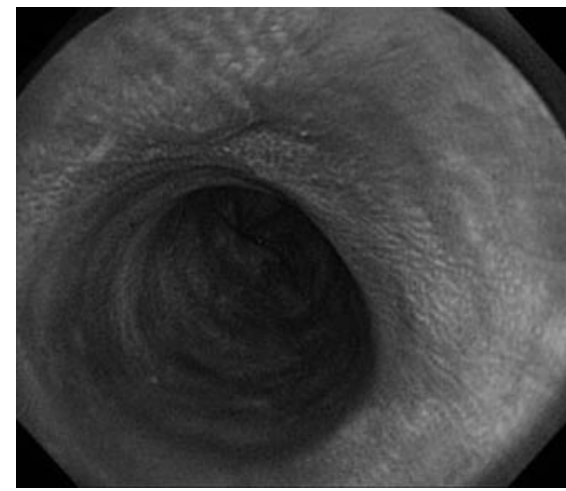

Figure 1. Standard esophagoscopy (A) and chromoendoscopy with iodine (B, unstained area with a pink-color sign) show that an esophageal squamous cell carcinoma (ESCC) of the lower esophagus was recurrent in a male 69 years of age, 12 months after achieving complete response with chemoradiotherapy consisting of 5-fluorouracil and cisplatin. Magnified endoscopic observation with narrow band imaging (NBI) shows an abnormal microvascular architectural pattern, indicating definitive ESCC (C). Photodynamic therapy (PDT), which started with the intravenous administration of $2 \mathrm{mg}$ / $\mathrm{kg}$ of Photofrin, was followed by irradiation with a $630-\mathrm{nm}$ excimer dye laser 48 and $72 \mathrm{~h}$ later. The endoscopic view shows the necrotic and ischemic injury in the irradiated region 7 days after PDT at a total of $1065 \mathrm{~J}$ (D). Follow-up standard esophagoscopy (E) and chromoendoscopy (F) show disappearance of the recurrent ESCC, and the intra-epithelial papillary capillary loop returned to a non-tumor pattern on magnifying endoscopy with NBI (G).

effect was observed with the $\mathrm{IC}_{50}$ of Photofrin at $0.83 \mu \mathrm{g} / \mathrm{ml}$ for the same PDT setting. The $\mathrm{IC}_{50}$ at a laser power of $5 \mathrm{~J} / \mathrm{cm}^{2}$ decreased 3-fold (3.3 $\mu \mathrm{g} / \mathrm{ml})$ in the presence of $9 \mu \mathrm{M}$ of CDDP. When PDT was combined with $10 \mu \mathrm{g} / \mathrm{ml}$ of Photofrin at a laser power of $5 \mathrm{~J} / \mathrm{cm}^{2}$ with the two chemotherapeutic agents at various concentrations, the $\mathrm{IC}_{50}$ s were substantially reduced from 3.5 and $9.0 \mu \mathrm{M}$ to 0.75 and $1.2 \mu \mathrm{M}$ for CDDP and 5-FU, respectively. 


\section{Discussion}

In the present study, $12(80 \%)$ of the 15 patients with superficial ESCC achieved CR with Photofrin-mediated PDT. The group consisted of no less than 13 patients with recurrent ESCC after CRT. In a similar setting of ESCC cases, Yano et al reported that the CR rate was $62 \%$ (8 of 13 patients) by salvage PDT after CRT failure. In their study, the overall survival rate 1 year after salvage PDT was $68.4 \%$ (14). In this study, 13 of the 15 patients were alive, and 11 were disease-free at the median follow-up period of 23 months. Two patients succumbed due to metastatic disease although no local recurrence of ESCC was noted after PDT. Hattori et al reported that the overall survival rate of patients treated by salvage EMR for locoregional failure after CRT was 56\% at 3 years (5). On the other hand, previous data showed that overall 3-year survival for patients with non-CR with definitive CRT was no more than 6\% (3). Collectively, the results suggest that local treatment by endoscopic modalities such as EMR and PDT is a treatment option for superficial ESCC without metastasis.

From a technical point of view, PDT appears to be superior to EMR. When the corresponding lesion exhibits ulceration, severe fibrosis, or stenosis, salvage EMR is difficult or impossible to perform (6). Little information is available on clinical outcomes of ESD in the salvage setting, although ESD permits the removal of esophageal epithelial neoplasms en bloc, irrespective of size (7). Nevertheless, in ESCC cases with invasion in the submucosal layer, ESD/EMR cannot be used due to its lack of curative potential. Even in such cases, PDT may yield relatively high CR rates, as indicated by the present and previous data (14). Surgical resection has been considered a salvage modality for these patients. However, Swisher et al reported that patients treated with salvage esophagectomy had a significantly higher incidence of anastomotic leaks (39 vs. $7 \%$ ) and a longer hospital stay ( 29 vs. 18 days) than those treated with planned esophagectomy (4). ESCC cases treated with CRT may occasionally be unresectable due to concomitant diseases (13). Thus, PDT offers an attractive alternative for patients with ESCC tumors without metastasis who would not otherwise be referred to a surgeon for treatment. Moreover, in selected patients with primary superficial ESCC, PDT as definitive therapy may avoid the risks associated with esophageal resection (13).

PDT was performed safely in the present and previous studies, and the majority of complications were manageable with medical treatment $(13,14)$. However, it is of clinical relevance that life-threatening complications are rarely noted. An esophagotracheal fistula may develop with PDT even in naïve early esophageal cancer cases; the reported incidence is $6.5 \%$ (14). Similarly, severe mediastinitis and pericardial effusion were previously documented following salvage PDT $(13,14)$. Possible reasons for the complications include radiation-induced esophageal damage and heart disease as well as transmural necrosis potentiated by PDT. Stricture formation at the irradiated esophageal site was common following PDT, occurring in $20-42 \%$ of cases. The majority of patients who underwent 3 or more PDT treatments developed a stricture requiring dilation, and the number of attempts varied (range 1-20, mean 2.7) (13).
Nevertheless, the indications for prophylactic pneumatic dilation warrant further evaluation.

Oral S-1, the 5-FU modulating drug, was administered to patients with submucosal ESCC with a potential risk of metastatic disease in the present study. The patients were still alive without significant complications despite their shortterm follow-up. Keeley et al applied chemotherapy and/or radiation therapy in 16 (32\%) of 50 patients with Barrett's highgrade dysplasia and esophageal cancer, varying from T1 to T3 tumor, to supplement primary PDT (13). Of the 16 patients who received concurrent chemoradiation, 6 remained alive at a mean interval of 16 months. Four of the patients who received PDT/chemo/radiation showed no evidence of disease at a mean interval of 13 months, and 2 were alive with disease. Conversely, all 10 patients succumbing to the disease had local recurrence. Due to superior survival and local control, esophagectomy may remain the preferred treatment for patients without physiological impairment. Nevertheless, the preliminary data on chemotherapy using the single fluorouracil along with PDT prompted us to investigate their combined effects in vitro. 5-FU and CDDP are widely used in chemotherapy regimens against various types of cancers. Combined chemotherapy consisting of the two key drugs has been a representative standard regimen against ESCC (19). Therefore, we focused on 5-FU and CDDP to enhance the cytotoxicity of Photofrin-mediated PDT in cellular experiments.

Upon activation by the special wavelength light, such Photofrin-derived photosensitizers undergo photochemical reactions to transfer electrons or hydrogen or to form singlet oxygen and generate excessive ROS $(11,20)$. This leads to oxidative damage to proteins, lipids and DNA, resulting in apoptotic or necrotic cell death $(11,20,21)$. In line with this theoretical concept, the patients who achieved CR had a significant increase in circulating ROS levels following PDT, which was not observed in non-CR cases. Our in vitro study showed that the combination of Photofrin-mediated PDT and 5-FU or CDDP resulted in a significantly lower cell survival than the single-mode treatment. Notably, with combined treatment, each $\mathrm{IC}_{50}$ dosage was significantly decreased; in particular, much lower concentrations of Photofrin and 5-FU were required to obtain sufficient cell killing and vice versa. Promising results using similar or other chemotherapeutic drugs have been reported in combination with PDT $(17,22,23)$. The synergistic effects of the two treatments may suggest diverse potential mechanisms for cellular death, one directly associated with PDT action and the other associated with each cytotoxic drug (17,22-24). Based on the in vitro results and our study, it is evident that more studies are warranted to determine the manner in which chemotherapeutic drugs and PDT interact and can be combined in order to result in increased cell killing with reduced side effects.

In conclusion, PDT demonstrated acceptable short-term outcomes, feasible curative properties, and safety for the treatment of superficial ESCC. Although further long-term follow-up studies are required, PDT is a promising treatment option for selected ESCC cases, particularly local recurrence following CRT. Combination therapy with PDT and 5-FU or CDDP may result in enhanced cytotoxic effects on ESCC, possibly reducing the effective dosage of each drug and decreasing side effects. 


\section{References}

1. Cooper JS, Guo MD, Herskovic A, et al: Chemoradiotherapy of locally advanced esophageal cancer: long-term follow-up of a prospective randomized trial (RTOG 85-01). Radiation Therapy Oncology Group. JAMA 281: 1623-1627, 1999.

2. Herskovic A, Martz K, Al-Sarraf M, et al: Combined chemotherapy and radiotherapy compared with radiotherapy alone in patients with cancer of the esophagus. N Engl J Med 326: $1593-1598,1992$

3. Ishikura S, Nihei K, Ohtsu A, et al: Long-term toxicity after definitive chemoradiotherapy for squamous cell carcinoma of the thoracic esophagus. J Clin Oncol 21: 2697-2702, 2003.

4. Swisher SG, Wynn P, Putnam JB, et al: Salvage esophagectomy for recurrent tumors after definitive chemotherapy and radiotherapy. J Thorac Cardiovasc Surg 123: 175-183, 2002.

5. Hattori S, Muto M, Ohtsu A, et al: EMR as salvage treatment for patients with locoregional failure of definitive chemoradiotherapy for esophageal cancer. Gastrointest Endosc 58: 65-70, 2003 .

6. Gotoda T, Kondo H, Ono H, et al: A new endoscopic mucosal resection procedure using an insulation-tipped electrosurgical knife for rectal flat lesions: report of two cases. Gastrointest Endosc 50: 560-563, 1999.

7. Isomoto $\mathrm{H}$ and Yamaguchi N: Endoscopic submucosal dissection in the era of proton pump inhibitors. J Clin Biochem Nutr 44 205-211, 2009

8. Fujishiro M, Kodashima S, Goto O, et al: Endoscopic submucosal dissection for esophageal squamous cell neoplasms. Dig Endosc 21: 109-115, 2009.

9. IsomotoH, Nishiyama H, Yamaguchi N, et al: Clinicopathological factors associated with clinical outcomes of endoscopic submucosal dissection for colorectal epithelial neoplasms. Endoscopy 41: 679-683, 2009.

10. Wolfsen HC: Uses of photodynamic therapy in premalignan and malignant lesions of the gastrointestinal tract beyond the esophagus. J Clin Gastroenterol 39: 653-664, 2005.

11. Robertson CA, Evans DH and Abrahamse H: Photodynamic therapy (PDT): a short review on cellular mechanisms and cancer research applications for PDT. J Photochem Photobiol B 96: 1-8, 2009.

12. Hahn SM, Putt ME, Metz J, et al: Photofrin uptake in the tumor and normal tissues of patients receiving intraperitoneal photodynamic therapy. Clin Cancer Res 12: 5464-5470, 2006.
13. Keeley SB, Pennathur A, Gooding W, Landreneau RJ, Christie NA and Luketich J: Photodynamic therapy with curative intent for Barrett's esophagus with high grade dysplasia and superficial esophageal cancer. Ann Surg Oncol 14: 2406-2410, 2007.

14. Yano T, Muto M, Minashi K, Ohtsu A and Yoshida S: Photodynamic therapy as salvage treatment for local failures after definitive chemoradiotherapy for esophageal cancer. Gastrointest Endosc 62: 31-36, 2005.

15. Hayashi I, Morishita Y, Imai K, Nakamura M, Nakachi K and Hayashi T: High-throughput spectrophotometric assay of reactive oxygen species in serum. Mutat Res 63: 55-61, 2007.

16. Fujii M, Kochi M and Takayama T: Recent advances in chemotherapy for advanced gastric cancer in Japan. Surg Today 40: 295-300, 2010

17. Nonaka M, Ikeda $\mathrm{H}$ and Inokuchi T: Effect of combined photodynamic and chemotherapeutic treatment on lymphoma cells in vitro. Cancer Lett 184: 171-178, 2002.

18. Veinot JP and Edwards WD: Pathology of radiation-induced heart disease: a surgical and autopsy study of 27 cases. Hum Pathol 27: 766-773, 1996.

19. Ohtsu A, Yoshida S and Saijo N: Disparities in gastric cancer chemotherapy between the East and West. J Clin Oncol 24: 2188-2196, 2006.

20. Plaetzer K, Krammer B, Berlanda J, Berr F and Kiesslich T: Photophysics and photochemistry of photodynamic therapy: fundamental aspects. Lasers Med Sci 24: 259-268, 2009.

21. Wang J and Yi J: Cancer cell killing via ROS: to increase or decrease, that is the question. Cancer Biol Ther 7: 1875-1884, 2008.

22. Park S, Hong SP, Oh TY, Bang S, Chung JB and Song SY: Paclitaxel augments cytotoxic effect of photodynamic therapy using verteporfin in gastric and bile duct cancer cells. Photochem Photobiol Sci 7: 769-774, 2008.

23. Zimmermann A, Walt H, Haller U, Baas P and Klein SD: Effects of chlorin-mediated photodynamic therapy combined with fluoropyrimidines in vitro and in a patient. Cancer Chemother Pharmacol 51: 147-154, 2003.

24. Takahira K, Sano M, Arai $\mathrm{H}$ and Hanai $\mathrm{H}$ : Apoptosis of gastric cancer cell line MKN45 by photodynamic treatment with Photofrin. Lasers Med Sci 19: 89-94, 2004. 